\title{
PRODUCT FORMS BASED ON BACKWARD TRAFFIC EQUATIONS
}

\author{
RICHARD J. BOUCHERIE,* CWI, Amsterdam
}

\begin{abstract}
This paper introduces a new form of local balance and the corresponding product-form results. It is shown that these new product-form results allow capacity constraints at the stations of a queueing network without reversibility assumptions and without special blocking protocols. In particular, exact product-form results for heavily loaded queueing networks are obtained.
\end{abstract}

LOCAL BALANCE; BLOCKING; QUEUEING NETWORK

AMS 1991 SUBJECT CLASSIFICATION: PRIMARY 60K25

SECONDARY $90 \mathrm{~B} 22$

\section{Introduction}

Queueing networks are widely used in computer performance evaluation, telecommunications and manufacturing. In particular, product-form queueing networks allow us to obtain accessible expressions for performance measures of interest. A major restriction of product-form queueing networks is that they do not allow the introduction of capacity constraints, unless very restrictive assumptions are made on the routing process of the customers.

Product-forms for queueing networks are based on the traffic equations. It is the structure of the state-dependent traffic equations that introduces the restrictive assumptions necessary to obtain product-form results when upper boundaries for the number of customers at stations are introduced. In contrast, lower boundaries for the number of customers at the queues can be chosen almost arbitrarily without destroying the productform result. This is also a consequence of the form of the traffic equations.

The standard traffic equations are the equilibrium equations for a queueing network containing a single customer moving from one queue to another according to a transition matrix of routing probabilities. As such these traffic equations are the Kolmogorov forward equations for the Markov chain describing the behaviour of a single customer in the queueing network. For this Markov chain one might also consider the Kolmogorov backward equations. A new type of traffic equations, closely related to the backward

Received 5 February 1993; revision received 1 November 1994.

* Present address: Universiteit van Amsterdam, Department of Econometrics, Roetersstraat 11, 1018 WB Amsterdam, The Netherlands.

Research carried out partly while the author was with the Department of Econometrics, Vrije Universiteit, Amsterdam, and partly while the author was ERCIM fellow at CWI, Amsterdam. Supported by the European Grant BRA-QMIPS of CEC DG XIII. 
equations, will be referred to as the backward traffic equations, and are the basis for the analysis and results presented in this paper.

This paper shows that product forms can be derived based on the backward traffic equations. These product forms are similar to the product forms obtained for 'standard' queueing networks such as Jackson networks [8], Gordon-Newell networks [5], BCMP networks [1], and Kelly-Whittle networks [9], [11]. The major difference between product forms based on the backward traffic equations and product forms based on the 'standard' traffic equations is that upper boundaries for the number of customers at the stations can be introduced without restrictive conditions on the routing process when the backward traffic equations are used, whereas lower boundaries introduce restrictions on the routing process. In particular, this allows us to obtain product-form results for heavily loaded queueing networks without restrictions such as blocking protocols. In addition to the results that can be obtained via job-hole duality, open networks are included in the formalism and blocking protocols are introduced to model lower boundaries.

In Section 2 we discuss the backward traffic equations and the corresponding form of local balance that is used to prove the product-form result. In Section 3 we investigate the structure of the backward traffic equations in detail. In particular, blocking protocols are investigated. Furthermore, Section 3 presents the generalisation to more general traffic equations. This generalisation is straightforward, and therefore postponed to this last section so as not to distract the reader from the ideas behind the backward traffic equations and corresponding product-form results. Finally, product forms are discussed for heavily loaded queueing networks. The paper concludes with a discussion of the results in Section 4.

\section{Model and product-form result}

Consider a continuous-time queueing network consisting of $N$ queues, or stations labelled $1, \cdots, N$. Assume that the queueing network can be represented by a stable, regular, continuous-time Markov chain $X=\{X(t), t \geqq 0\}$ at state space $S \subseteq \mathbb{N}_{0}^{N}$. A state $\boldsymbol{n}=\left(n_{1}, \cdots, n_{N}\right) \in S$ is a vector with components $n_{i} \in \mathbb{N}_{0}$ denoting the number of customers at queue $i, i=1, \cdots, N$.

Let $q\left(\boldsymbol{n}, \boldsymbol{n}^{\prime}\right)$ denote the transition rate from state $\boldsymbol{n}$ to state $\boldsymbol{n}^{\prime}$. As transitions correspond to customers routing among the queues, we have $q\left(\boldsymbol{n}, \boldsymbol{n}^{\prime}\right)=0$ unless $\boldsymbol{n}^{\prime}=$ $\boldsymbol{n}-\boldsymbol{e}_{i}+\boldsymbol{e}_{j}, i, j=0, \cdots, N$, where $\boldsymbol{e}_{i}$ denotes the $i$ th unit vector, and $\boldsymbol{e}_{0}=0$. A transition $\boldsymbol{n} \rightarrow \boldsymbol{n}-\boldsymbol{e}_{i}+\boldsymbol{e}_{j}$ corresponds to a customer routing from station $i$ to station $j$, and for notational convenience station 0 represents the outside.

Assume that $X$ is irreducible at $S$ and that $X$ possesses a stationary or equilibrium distribution $\pi=(\pi(n), n \in S)$ at $S$. Then $\pi$ is the unique solution of the global balance equations (Whittle [11])

$$
\sum_{\boldsymbol{n}^{\prime} \in S}\left\{\pi(\boldsymbol{n}) q\left(\boldsymbol{n}, \boldsymbol{n}^{\prime}\right)-\pi\left(\boldsymbol{n}^{\prime}\right) q\left(\boldsymbol{n}^{\prime}, \boldsymbol{n}\right)\right\}=0, \quad \boldsymbol{n} \in S .
$$

An immediate consequence of the transition structure of $X$ is that the global balance equations can be written as 


$$
\sum_{i, j=0}^{N}\left\{\pi(\boldsymbol{n}) q\left(\boldsymbol{n}, \boldsymbol{n}-\boldsymbol{e}_{i}+\boldsymbol{e}_{j}\right)-\pi\left(\boldsymbol{n}-\boldsymbol{e}_{i}+\boldsymbol{e}_{j}\right) q\left(\boldsymbol{n}-\boldsymbol{e}_{i}+\boldsymbol{e}_{j}, \boldsymbol{n}\right)\right\}=0, \quad \boldsymbol{n} \in S
$$

Product-form results for queueing networks are based on local balance, balancing for each queue separately the flow out of a state due to the departure of customers and the flow into that state due to the arrival of customers. A distribution $\pi$ satisfies local balance if for $i=0, \cdots, N$

$$
\sum_{j=0}^{N}\left\{\pi(\boldsymbol{n}) q\left(\boldsymbol{n}, \boldsymbol{n}-\boldsymbol{e}_{i}+\boldsymbol{e}_{j}\right)-\pi\left(\boldsymbol{n}-\boldsymbol{e}_{i}+\boldsymbol{e}_{j}\right) q\left(\boldsymbol{n}-\boldsymbol{e}_{i}+\boldsymbol{e}_{j}, \boldsymbol{n}\right)\right\}=0, \quad \boldsymbol{n} \in S .
$$

A distribution $\pi$ that satisfies (2.2) is the equilibrium distribution because (2.2) is the decomposition of (2.1) into a set of equations for each $i$ separately.

Similar to the decomposition of (2.1) into a set of equations for each $i,(2.1)$ can also be decomposed into a set of equations for each $j$. This gives a form of local balance that we will refer to as backward local balance equating for each queue separately the flow (probability flux) out of a state due to the arrival of customers and the flow (probability flux) into that state due to the departure of customers from that queue (see Figure 1). A distribution $\pi^{\mathrm{b}}$ satisfies backward local balance if for $j=0, \cdots, N$

$$
\sum_{i=0}^{N}\left\{\pi^{\mathrm{b}}(\boldsymbol{n}) q\left(\boldsymbol{n}, \boldsymbol{n}-\boldsymbol{e}_{i}+\boldsymbol{e}_{j}\right)-\pi^{\mathrm{b}}\left(\boldsymbol{n}-\boldsymbol{e}_{i}+\boldsymbol{e}_{j}\right) q\left(\boldsymbol{n}-\boldsymbol{e}_{i}+\boldsymbol{e}_{j}, \boldsymbol{n}\right)\right\}=0, \quad \boldsymbol{n} \in S .
$$

At first glance, (2.2) and (2.3) seem to express a similar form of local balance. However, note that in general $\pi \neq \pi^{\mathrm{b}}$, and that a solution $\pi$ to (2.2) may exist while a solution $\pi^{\mathrm{b}}$ to (2.3) does not exist and vice versa. This is an immediate consequence of the restrictive nature of the assumption that a process satisfies (backward) local balance.

Below we present sufficient conditions for the existence of a solution to the backward local balance equations. These conditions are similar to the conditions sufficient to find a solution to the local balance equations. Note that the solution can be the same only if $\boldsymbol{X}$ is reversible.

Assume that the transition rates have the form

$$
q\left(\boldsymbol{n}, \boldsymbol{n}-\boldsymbol{e}_{i}+\boldsymbol{e}_{j}\right)=\frac{\phi\left(\boldsymbol{n}+\boldsymbol{e}_{j}\right)}{\psi(\boldsymbol{n})} p_{i j}\left(\boldsymbol{n}+\boldsymbol{e}_{j}\right)
$$

where $\phi, \psi$, and $p_{i j}$ are arbitrary functions such that $\psi(\boldsymbol{n})>0$ for all $\boldsymbol{n} \in S$. We have the following result.

Theorem 2.1. Assume that a positive solution $\left\{d_{i}\right\}_{i=1}^{N}$ exists for the backward state dependent traffic equations for $j=0, \cdots, N$,

$$
\sum_{i=0}^{N}\left\{d_{j} p_{i j}\left(\boldsymbol{n}+\boldsymbol{e}_{j}\right)-d_{i} p_{j i}\left(\boldsymbol{n}+\boldsymbol{e}_{j}\right)\right\}=0, \quad d_{0}=1 .
$$

Then $X$ has a unique equilibrium distribution 


$$
\pi(\boldsymbol{n})=B \psi(\boldsymbol{n}) \prod_{k=1}^{N}\left(\frac{1}{d_{k}}\right)^{n_{k}}, \quad n \in S,
$$

where

$$
B^{-1}=\sum_{n \in S} \psi(\boldsymbol{n}) \prod_{k=1}^{N}\left(\frac{1}{d_{k}}\right)^{n_{k}}<\infty,
$$

and $\pi$ satisfies backward local balance (2.3).

Proof. Insertion of (2.4) and (2.6) in the backward local balance equations gives

$$
\begin{aligned}
& \sum_{i=0}^{N}\left\{\pi(n) q\left(n, n-e_{i}+e_{j}\right)-\pi\left(n-e_{i}+e_{j}\right) q\left(n-e_{i}+e_{j}, n\right)\right\} \\
& \quad=B \phi\left(n+e_{j}\right) \prod_{k=1}^{N}\left(\frac{1}{d_{k}}\right)^{n_{k}+\delta_{k j}} \sum_{i=0}^{N}\left\{d_{j} p_{i j}\left(n+e_{j}\right)-d_{i} p_{j i}\left(n+e_{j}\right)\right\},
\end{aligned}
$$

and (2.5) completes the proof.

Remark 2.2

1. The structure of the transition rates $(2.4)$ is similar to the structure used in the literature on product-form queueing networks (Boucherie and van Dijk [3], Serfozo [10])

$$
q\left(\boldsymbol{n}, \boldsymbol{n}-\boldsymbol{e}_{i}+\boldsymbol{e}_{j}\right)=\frac{\gamma\left(\boldsymbol{n}-\boldsymbol{e}_{i}\right)}{\psi(\boldsymbol{n})} p_{i j}\left(\boldsymbol{n}-\boldsymbol{e}_{i}\right) .
$$

The main difference is the dependence on $n+e_{j}$ in (2.4) and on $n-e_{i}$ in (2.7). This difference is related to the structure of the local balance equations. From (2.3) and the proof of Theorem 2.1 we see that $\phi\left(n+\boldsymbol{e}_{j}\right)$ appears as a constant for these local balance equations, whereas from (2.2) we similarly see that $\gamma\left(\boldsymbol{n}-\boldsymbol{e}_{i}\right)$ is a constant for this set of local balance equations. This is the explanation of the choice $(2.4)$ for the transition rates.

2. The backward traffic equations have a structure related to the backward equations for the single customer process. This is best illustrated when we consider the stateindependent routing version of (2.5):

$$
\sum_{i=0}^{N}\left\{p_{i j} d_{j}-p_{j i} d_{i}\right\}=0, \quad d_{0}=1
$$

The standard traffic equations for a queueing network with routing probabilities $p_{i j}$ are

$$
\sum_{j=0}^{N}\left\{c_{i} p_{i j}-c_{j} p_{j i}\right\}=0, \quad c_{0}=1
$$

The difference between (2.8) and (2.9) is that $d_{i}$ appears on the other side of the routing probabilities. Note that (2.8) does not correspond to the backward equations of the single-customer process. The backward equations are $\Sigma_{i=0}^{N}\left\{p_{j i} d_{i}-p_{j i} d_{j}\right\}=0$. Therefore, in contrast with the results for product-form queueing networks where the existence 
of a solution to the traffic equations (2.9) follows from the Perron-Frobenius theorem, for the backward process a solution to (2.8) is not guaranteed. In Section 3.1 we present special cases in which a solution to (2.8) exists. In particular, in Section 3.1 we transform (2.8) into an equation of the form (2.9) with a transformation of the routing matrix $p_{i j}$ into a probability matrix $\bar{p}_{i j}$. In Section 3.2 we present blocking results that are such that the solution for $(2.8)$ is a solution for (2.5) too.

3. As a consequence of the form of the transition rates (2.4), and the traffic equations (2.5), the equilibrium distribution has a product form similar to the standard product form for queueing networks. The ideas behind the product form (2.6) are different: it is derived on the basis of the backward local balance equations. As discussed below, this allows us to introduce capacity constraints at the stations without additional conditions on the transition structure. In particular, backward local balance allows us to derive product-form results for heavily loaded networks without constraints on the transition rates such as blocking protocols. Related results for heavily loaded networks are obtained by Gordon and Newell [6] and Hordijk and van Dijk [7]. In these references networks with transition rates (2.7) are analysed via methods related to backward local balance as defined above. Since the transition rates (2.7) are not compatible with backward local balance, the networks of these references are allowed to contain single server queues only.

\section{Examples and extensions}

In contrast with standard product-form queueing networks, where a positive solution for the traffic equations (2.9) is guaranteed by the Perron-Frobenius theorem, the backward traffic equations (2.8) will not always have a positive solution. Section 3.1 presents some special cases in which a solution for the backward traffic equations is guaranteed. In Section 3.2 we will investigate the behaviour of the state-dependent traffic equations at the boundary of the state space, and in Section 3.3 we generalise the result of Theorem 2.1 to state-dependent solutions of the traffic equations. Finally, in Section 3.4 we use the product-form results of Gordon and Newell [6] and Hordijk and van Dijk [7] for heavily loaded networks of single-server queues to illustrate our results.

3.1. State-independent routing. Consider the state-independent backward traffic equations (2.8), a special case of (2.5) in which the state is removed from the routing function. A solution for (2.8) will in general not satisfy (2.5) because of conditions imposed by the boundary of the state space $S$, but in most applications a solution of (2.8) is the basis of a solution for (2.5). This is discussed in Section 3.2.

If $P=\left(p_{i j}\right)$ is doubly stochastic, that is

$$
\sum_{j=0}^{N} p_{i j}=1 \text { and } \sum_{i=0}^{N} p_{i j}=1 \text {, }
$$

then the backward traffic equations are equivalent to the backward equations for the single customer Markov chain. Thus if this Markov chain is irreducible the Perron- 
Frobenius theorem guarantees a unique solution $d_{i}=1$ for all $i$. This is not a very interesting case.

It is not assumed that the coefficients $p_{i j}$ figuring in the backward traffic equations are routing probabilities, that is $\sum_{j=0}^{N} p_{i j}=h_{i} \neq 1$ is possible. The following choices, each with $\bar{p}_{i j}$ the routing probabilities for a single-customer Markov chain, exploit the possibility that $h_{i} \neq 1$ is allowed. In these examples the routing function is chosen such that the Perron-Frobenius theorem for the single customer process can be used to guarantee a unique solution for (2.8).

Let $\left\{\bar{c}_{i}\right\}_{i=1}^{N}$ be the unique solution to $\sum_{j=0}^{N}\left\{\bar{c}_{i} \bar{p}_{i j}-\bar{c}_{j} \bar{p}_{j i}\right\}=0, \bar{c}_{0}=1$, then

$$
\begin{array}{ll}
\text { if } p_{i j}=\bar{c}_{i} \bar{c}_{j} \bar{p}_{i j} & \text { we have } d_{i}=1 / \bar{c}_{i}, \\
\text { if } p_{i j}=\bar{c}_{j}^{2} \bar{p}_{j i} & \text { we have } d_{i}=1 / \bar{c}_{i}, \\
\text { if } p_{i j}=\left(\bar{c}_{i} / \bar{c}_{j}\right) \bar{p}_{i j} & \text { we have } d_{i}=\bar{c}_{i}, \\
\text { if } p_{i j}=\bar{p}_{j i} & \text { we have } d_{i}=\bar{c}_{i},
\end{array}
$$

and in each of these cases $\left\{d_{i}\right\}_{i=1}^{N}$ is unique. The first two cases correspond to the Markov chain $X$ describing customers, whereas the last two cases correspond to $X$ describing vacancies. A discussion on this interpretation can be found in Boucherie [2].

Finally, if the routing probabilities $\bar{p}_{i j}$ are such that a solution for the detailed balance equations $\bar{c}_{i} \bar{p}_{i j}=\bar{c}_{j} \bar{p}_{j i}$ exists, then for $p_{i j}=\bar{p}_{i j}$ we have that $d_{i}=1 / \bar{c}_{i}$ satisfies $p_{i j} d_{j}=p_{j i} d_{i}$.

The above examples make use of the Perron-Frobenius theorem for a related Markov chain with routing probabilities $\bar{p}_{i j}$. In general we have to solve $(2.8)$ to see whether or not a positive solution exists.

To provide some insight in the solutions presented above, assume that the solutions for (2.8) presented above satisfy (2.5) too. Consider the cases $p_{i j}^{1}=\bar{c}_{i} \bar{c}_{j} \bar{p}_{i j}$ and $p_{i j}^{2}=$ $\left(\bar{c}_{i} / \bar{c}_{j}\right) \bar{p}_{i j}$ discussed above. The transition rates (2.4) corresponding to these cases are

$$
q^{1}\left(\boldsymbol{n}, \boldsymbol{n}-\boldsymbol{e}_{i}+\boldsymbol{e}_{j}\right)=\frac{\phi\left(\boldsymbol{n}+\boldsymbol{e}_{j}\right)}{\psi(\boldsymbol{n})} \bar{c}_{i} \bar{c}_{j} \bar{p}_{i j}, \quad q^{2}\left(\boldsymbol{n}, \boldsymbol{n}-\boldsymbol{e}_{i}+\boldsymbol{e}_{j}\right)=\frac{\phi\left(\boldsymbol{n}+\boldsymbol{e}_{j}\right)}{\psi(\boldsymbol{n})}\left(\bar{c}_{i} / \bar{c}_{j}\right) \bar{p}_{i j},
$$

and $X$ has a unique equilibrium distribution

$$
\pi^{1}(\boldsymbol{n})=B \psi(\boldsymbol{n}) \prod_{k=1}^{N} \bar{c}_{k}^{n_{k}} \quad \text { and } \quad \pi^{2}(\boldsymbol{n})=B \psi(\boldsymbol{n}) \prod_{k=1}^{N}\left(1 / \bar{c}_{k}\right)^{n_{k}}
$$

Observe that $\pi^{1}$ satisfies standard local balance (2.2) for the process with transition rates (2.7), and that $\pi^{1}$ therefore is the equilibrium distribution for the process with transition rates (2.7) too. In contrast, we cannot select functions $\phi, \psi$, and $\gamma$ such that $\pi^{2}$ satisfies standard local balance (2.2). Therefore, the product form solutions for the backward local balance equations (2.3) found in Theorem 2.1 are in general different from the product form results based on standard local balance (2.2).

3.2. Blocking protocols (for open queueing networks). In this section we discuss the behaviour of $X$ at boundaries. In particular, blocking protocols are presented that preserve the product form equilibrium distribution. As will be shown, due to the 
structure of the local balance equations, upper limit blocking corresponding to capacity constraints can be introduced without restrictions, but lower bounds require some assumptions on the $p_{i j}$.

For simplicity, assume that $p_{i j}\left(n+e_{j}\right)$ is state-independent, except for states near the boundary of $S$, that is for all $n, j$ such that $n+e_{j} \in S$

$$
p_{i j}\left(n+e_{j}\right)=p_{i j} b_{i j}\left(n+e_{j}\right),
$$

where $b_{i j}\left(n+e_{j}\right)=1$ away from the boundary in a way presented below, and $p_{i j}$ is such that a positive solution exists for (2.8).

If (2.8) admits a solution for $p_{i j} d_{j}-p_{j i} d_{i}=0$, then for all $i, j, n+e_{j}$ we may set $b_{i j}\left(n+e_{j}\right)=1$. This result is similar to the result for reversible processes based on local balance (2.2).

Consider the case in which a 'reversible' solution for (2.8) does not exist. In Figure 1 for states in the interior of $S$ global balance and its decomposition into local balance (2.3) is depicted for a two-queue system. For comparison, the decomposition into local balance (2.2) is depicted too.

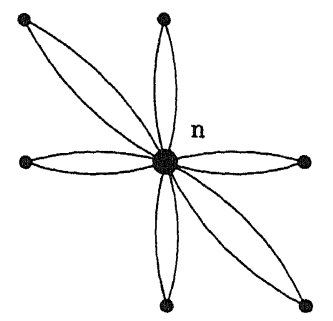

Global balance at $\mathbf{n}$

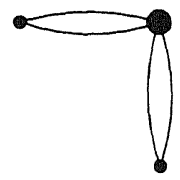

$j=0$

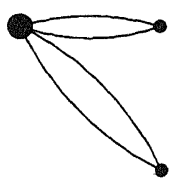

$j=1$

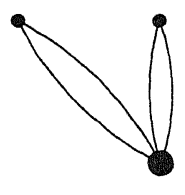

$j=2$

Backward local balance (2.3) at $\mathrm{n}$
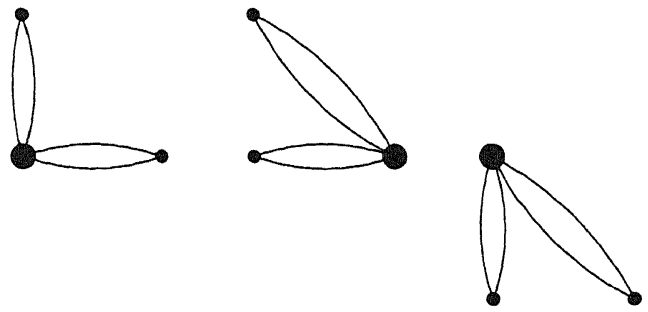

$i=0$

$i=1$

$i=2$

Standard local balance (2.2) at $\mathbf{n}$

Figure 1. Decomposition of global balance

For a product form equilibrium distribution to be a solution of (2.2) it is known that special blocking protocols must be used to change certain transitions near the boundary of $S$. For example, the stop protocol also referred to as communication blocking stops all queues except for a saturated queue to preserve product form (cf. Van Dijk [4]). A similar protocol is discussed below for (2.3). 
Consider the two-queue system of Figure 1. When we introduce a capacity constraint, say $n_{2} \leqq N_{2}$, this results in an upper bound for the state space. As a consequence, for $n$ such that $n_{2}=N_{2}$ transitions $n \leftrightarrow n+e_{2}$ and $n \leftrightarrow n-e_{1}+e_{2}$ cannot occur. This zorresponds to stopping of local balance for $j=2$, but local balance for $j=0$ and $j=1$ is not affected by this bound. Therefore, if $\left\{d_{i}\right\}_{i=1}^{2}$ is a solution for (2.8), then $\left\{d_{i}\right\}_{i=1}^{2}$ is a solution for (2.3) at the upper bound too. The same holds true for a queueing network of $N$ queues:

$$
\begin{aligned}
& \text { If }\left\{d_{i}\right\}_{i=1}^{N} \text { is a solution for (2.8) then }\left\{d_{i}\right\}_{i=1}^{N} \text { is also a solution for (2.3) at } \\
& \text { an upper bound without any restrictions on the transition rates. }
\end{aligned}
$$

When we introduce the same upper bound $n_{2} \leqq N_{2}$ for local balance (2.2), from Figure 1 ve see that local balance for $i=2$ is not affected, but that the upwards transitions for $=0$ and $i=1$ cannot occur. Therefore, for the solution $\left\{c_{i}\right\}_{i=1}^{2}$ for (2.9) to be a solution or (2.2) we must stop local balance for $i=0$ and $i=1$ at the boundary $n_{2}=N_{2}$.

This implies that local balance (2.3) is more suited for queueing networks with capacity constraints.

What we have gained at the upper bounds by using (2.3) instead of (2.2) is lost at the ower bounds. For queueing networks for which the equilibrium distribution satisfies 2.2) it is well known that a lower bound corresponding to zero customers at the queues an be introduced without any restrictions on the process. This lower bound is a natural estriction on the network. For queueing networks for which the equilibrium distribuion is found as a solution for (2.3) we have to introduce a blocking protocol to preserve re solution to (2.8). This can immediately be seen from Figure 1. If $n_{2}=0$ then ansitions $\boldsymbol{n} \leftrightarrow \boldsymbol{n}-e_{2}$ and $\boldsymbol{n} \leftrightarrow \boldsymbol{n}+e_{1}-e_{2}$ can no longer occur. Therefore local balance or $j=0$ and $j=1$ must be stopped, whereas local balance for $j=2$ is not affected. This locking protocol at the boundary $n_{2}=0$ can easily be formulated for queueing networks ith $N$ stations and states:

If a queue is empty only those transitions in which a customer moves to the empty queue are allowed.

he blocking protocol corresponds to a service system in which when a queue is empty ly a smart customer that selects the empty queue for its next service will be served, but in also be interpreted as a protocol that applies a load balancing procedure, balancing e load over the queues of the network.

From the above discussion we obtain that we have two blocking protocols that eserve product form. The first protocol (communication blocking) changes the behavur of the process at upper boundaries, and the second protocol (smart customer ocking) changes the behaviour of the process at lower boundaries. When a process ays close to a lower boundary with high probability (light traffic) the communication ocking protocol may be used, and we may use smart customer blocking when the ocess stays away from its lower boundaries with high probability (heavy traffic). In ction 3.4 below we consider the case in which the process stays away from its lower undaries with probability 1 . 
3.3. General traffic equations. The result of Section 2 can easily be generalised to general state-dependent solutions for the backward' state-dependent traffic equations (2.5). This gives the analogue of the general state-dependent traffic equations for queueing networks based on (2.2), as can for example be seen from Theorem 5.1 on p. 137 of Van Dijk [4]. The proof of the theorem is similar to the proof of Theorem 2.1 and is therefore omitted.

Theorem 3.1. Assume that a positive function $H(n)$ exists that satisfies

$$
\sum_{i=0}^{N}\left\{H(n) p_{i j}\left(n+e_{j}\right)-H\left(n-e_{i}+e_{j}\right) p_{j i}\left(n+e_{j}\right)\right\}=0,
$$

for all $n \in S, j=0, \cdots, N$ for which $\phi\left(n+\boldsymbol{e}_{j}\right)>0$. Then $\boldsymbol{X}$ has a unique equilibrium distribution

$$
\pi(n)=B \psi(n) H(n), \quad n \in S,
$$

where $B^{-1}=\Sigma_{n \in S} \psi(n) H(n)<\infty$, and $\pi$ satisfies backward local balance (2.3).

The above theorem generalises Theorem 2.1 in two ways. Firstly, the solution for the traffic equations is a state-dependent function. Secondly, (3.1) is required only if $\phi\left(n+e_{j}\right)>0$. Observe that the above theorem establishes a decomposition of the equilibrium distribution in a service part $\psi(\cdot)$ and a routing part $H(\cdot)$, and that these parts are linked via the normalisation constant $B$ only.

3.4. Heavily loaded networks. When we introduce a capacity constraint at each of the stations of a closed queueing network, in general product forms cannot be derived. As will be shown below, in lightly and heavily loaded systems product forms can still be obtained.

Let $S=\left\{\boldsymbol{m}: 0 \leqq n_{i} \leqq M_{i}<\infty\right\}$, that is assume that at most $M_{i}$ customers are allowed at queue $i, i=1, \cdots, N$. Let $M$ be the number of customers in the network and assume that all stations are single-server queues. The transition rates for this network are

$$
q\left(\boldsymbol{n}, \boldsymbol{n}-\boldsymbol{e}_{i}+\boldsymbol{e}_{j}\right)=\mu_{i} p_{i j} \mathbf{1}\left(n_{i}>0, n_{j}<M_{j}\right) .
$$

If $M \leqq \min _{i}\left\{M_{i}\right\}$, then blocking of customers does not occur, and the equilibrium distribution is of product form:

$$
\pi(\boldsymbol{n})=B \prod_{i=1}^{N}\left(c_{i} / \mu_{i}\right)^{n_{i}}, \quad n \in S, \quad\left(M \leqq \min _{i}\left\{M_{i}\right\}\right)
$$

where $\left\{c_{i}\right\}_{i=1}^{N}$ is a solution of the standard traffic equations (2.9), a result that can be concluded from the standard local balance equations (2.2).

Now assume that $M \geqq \sum_{i=1}^{N} M_{i}-\min _{i}\left\{M_{i}\right\}$; that is, assume that the network is heavily loaded such that no queue can empty. From Section 3.2 we obtain that the backward local balance equations do not impose restrictions on the process. Let $\left\{d_{i}\right\}_{i=1}^{N}$ be a solution of the backward traffic equations (2.8) for the process with routing function $\mu_{i} p_{i j}$ : 


$$
\sum_{i=1}^{N}\left\{\mu_{i} p_{i j} d_{j}-\mu_{j} p_{j i} d_{i}\right\}=0, \quad j=1, \cdots, N .
$$

Then

$$
\pi(\boldsymbol{n})=B \prod_{i=1}^{N}\left(1 / d_{i}\right)^{n_{i}}, \quad n \in S, \quad\left(M \geqq \sum_{i=1}^{N} M_{i}-\min _{i}\left\{M_{i}\right\}\right)
$$

is the equilibrium distribution at $S$, which can be concluded from Theorem 2.1 and backward local balance (2.3).

Observe that $\pi(n)=B \Pi_{i=1}^{N}\left(1 / d_{i}\right)^{n_{i}}$ does not satisfy (2.2). Due to blocking of customers at the stations a product form cannot be obtained from standard product form theory, but can easily be concluded from Theorem 2.1 .

When the network is cyclic we have $p_{i, i+1}=1$, and $d_{i}=1 / \mu_{i-1}, i=2, \cdots, N, d_{1}=$ $1 / \mu_{N}$, is the solution of (3.2). The equilibrium distribution is $\pi(n)=B \prod_{i=1}^{N} \mu_{i}^{n_{i+1}}$, where $n_{N+1} \stackrel{\text { def }}{=} n_{1}$. This result is obtained by Gordon and Newell [6] via job-hole duality arguments, and by Hordijk and van Dijk [7]. Observe that in both these references the results are restricted to networks of single-server queues. Therefore, Theorem 2.1 zeneralises the results of these references to queueing networks with arbitrary routing and more general service requirements.

\section{Discussion}

This paper has presented a new form of local balance for queueing networks and the sorresponding product-form results. These product-form results have a structure closely elated to standard product-form results. A major difference is the behaviour at soundaries of the state space. As is shown, the product-form queueing networks ntroduced in this paper allow us to introduce capacity constraints at the queues without :onditions on the transition rates. Smart customer blocking is introduced to preserve ocal balance at lower boundaries of the state space.

It is shown that product-form results for heavily loaded queueing networks can be ibtained from backward local balance. These results extend the product-form results ibtained by Gordon and Newell [6] for heavily loaded cyclic networks to networks with rbitrary topology under heavy load.

The product-form results introduced here may allow adequate approximations for jueueing networks in heavy traffic, as can be seen from the smart customer blocking irotocol at lower boundaries and the results related to job-hole duality. Additional esearch is necessary to investigate this possibility in detail. Furthermore, product-form esults for queueing networks and approximation schemes are available based on tandard local balance (2.2). Similar results seem to be possible based on backward local alance (2.3).

\section{ieferences}

[1] Baskett, F., Chandy, K. M., Muntz, R. R. and Palacios, F. G. (1975) Open, closed and lixed networks of queues with different classes of customers. Journal of the ACM 22, 248-260. 
[2] Boucherie, R. J. (1992) Product-form in queueing networks. Ph.D. thesis, Vrije Universiteit, Amsterdam (available from the author).

[3] Boucherie, R. J. AND VAN DiJK, N. M. (1991) Product forms for queueing networks with state dependent multiple job transitions. Adv. Appl. Prob. 23, 152-187.

[4] van DiJK, N. M. (1993) Queueing Networks and Product Forms: A Systems Approach. Wiley, New York.

[5] Gordon, W. J. AND Newell, G. F. (1967) Closed queueing systems with exponential servers. Operat. Res. 15, 254-265.

[6] Gordon, W. J. ANd Newell, G. F. (1967) Cyclic queueing systems with restricted length queues. Operat. Res. 15, 266-277.

[7] HoRdiJk, A. AND van Disk, N. M. (1981) Networks of queues with blocking. In Performance '81, ed. F. J. Kylstra, pp. 51-65. North-Holland, Amsterdam.

[8] JACKson, J. R. (1957) Networks of waiting lines. Operat. Res. 5, 518-521.

[9] Kelly, F. P. (1979) Reversibility and Stochastic Networks. Wiley, New York.

[10] Serfozo, R. F. (1989) Markovian network processes: congestion dependent routing and processing. Queueing Systems 5, 5-36.

[11] WhitTle, P. (1986) Systems in Stochastic Equilibrium. Wiley, New York. 